\title{
Converting an electronic calculator into a combination stopwatch-calculator
}

\author{
ALLEN H. WOLACH, PETER ROCCAFORTE, and SYLVIA N. VanBERSCHOT \\ Mlinois Institute of Technology, Chicago, Ilinois 60616 \\ and \\ MAUREEN A. McHALE \\ Northwestern State University of Louisiana, Natchitoches, Louisana 71457
}

\begin{abstract}
A method for adding electronic stopwatch capabilities to a calculator is described. An integrated circuit (NE555) is used for a time base. The circuit drives an opto-isolator or lightemitting diode that operates a photocell. The opto-isolator or photocell substitutes for a switch closure to increment the calculator in .10-sec intervals.
\end{abstract}

A pocket calculator can easily be converted to perform the functions of a stopwatch. Since the stopwatch can also be used as a calculator, it becomes an especially useful tool. A test administrator can time a subject on various tasks with the stopwatch and then total the subject's score with the calculator. The stopwatch feature can be added to a calculator for less than $\$ 5.00$.

\section{HARDWARE AND METHOD}

Select a calculator with a constant addition feature; i.e., repeatedly pushing the " + " key on some models, repeatedly adds a constant that is entered on the keyboard to the running total. The first step in the modification is the same as the modification for converting a calculator into a counter (Alexandrovich, 1975; Wolach, Roccaforte, \& Breuning, 1975). Since the keys on electronic calculators are switches, an external device (in this case, a photocell or the output from an optoisolator) can be used to substitute for switch closure. The keyboard for most calculators has flexible wires as they enter the calculator printed circuit board. Locate the wires for " + " (or " $=$ " on calculators that cumulate by pressing the "=" as opposed to the " + " key) by shorting two keyboard wires at a time until the calculator performs an addition. Wolach et al (1975) supply a table showing the wires for the "+" or "=" key on six representative calculators.

Figures 1 (schematic) and 2 (pictorial diagram) show the conversion for a typical calculator. The calculator in Figure 2 is a Novus 650, marketed by National Semiconductor, 2900 Semiconductor Way, Santa Clara, California. Wires 2 and 6 from the keyboard (looking from

Requests for reprints should be sent to Allen H.Wolach, Department of Psychology, Illinois Institute of Technology, Chicago, Illinois 60616 . the top of the printed circuit board with the number display visible) are for " + " in the Novus 650 .

The first step in the conversion provides a method for substituting a decrease in resistance for the switct closure that ordinarily operates the " $f$ " key of the calculator. The decrease in resistance is optically isolated from the timing circuitry that determines when the " + " key is activated. A light-emitting diode (LED) and a photocell are used in the first method for optically isolating the " $t$ " key from the timer.

Solder a photocell across the two wires for the " $t$ " (or " =") key. Select a photocell that has very low resistance when the light is on and very high resistance when the light is off (e.g., Clairex CL5M3). The photocell will act as a switch slosure to the " $f$ " (or "=") key

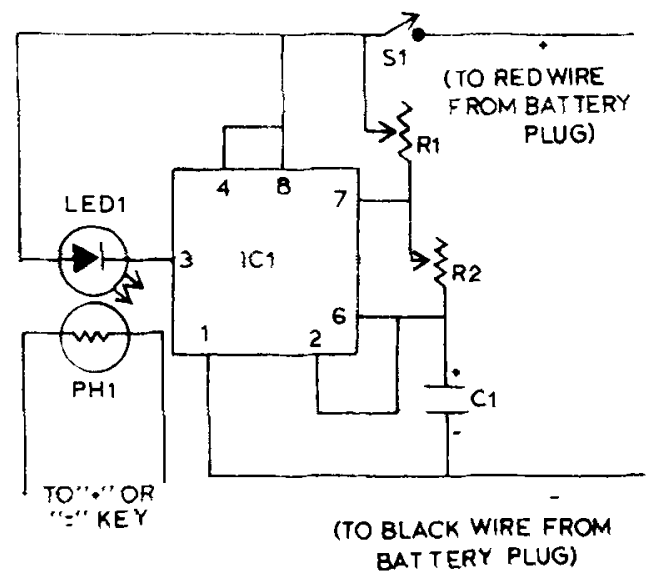

Figure 1. Circuit diagram for stopwatch provision. Capacitor $\mathrm{Cl}$ is an 100 microfarad, $10 \mathrm{~V}$, miniature electrolytic capacitor. Resistors R1 and R2 are $20,000 \mathrm{ohm}, .25-\mathrm{W}$, miniature potentiometers. The integrated circuit (ICI) is a Signetics NE555. Switch closure (S1) is provided by a silent miniature SPST slide switch. See text for details about the LED (e.g., Monsanto MV 5012) and photocell (PH1, e.g., Clairex, CL5M3). 
when a LED is turned on. The LED must work on the voltage supplied by the calculator's battery. A Monsanto MV5012 will work with batteries that produce $3 \mathrm{~V}$ or more. If the unit does not calculate when the photocell is blocked from light, the photocell has too little resistance in the lightoff situation. If the photocell does not operate the calculator in a dark room when a LED is brought into contact with the photocell, the photocell has too much resistance in the lighton situation. Connect a LED across a battery of the same voltage as the calculator's battery; the LED will only work if the positive side is connected to the positive side of the battery.

The second method for substituting a decrease in resistance for the switch closure uses an opto-isolator instead of the photocell-LED assembly. This method is simpler, but somewhat more expensive than the photocell-LED method. The opto-isolator produces an output analogous to the output of the photocell. The wires that go to to the LED in the photo-cell-LED method can be attached to the input of the opto-isolator. Select an opto-isolator that draws as little current as possible and provides a sufficient change in output resistance, e.g., the Vectec hermetic sealed vectrol, VTL1A2 for 6-V calculators or the Model VTL1A3 for 9.V calculators.

The timing circuit for the stopwatch calculator is a Signetics NE555 integrated circuit. The NE555 is inexpensive (less than \$1.50) and sufficiently stable for the stopwatch calculator. Since the NE555 is relatively voltage independent, the stopwatch does not lose accuracy as the stopwatch calculator battery runs down. The location of the additional components in calculators other than the Novus 650 will be determined by the location of the components in a given device. If a calculator (e.g., Craig 4511) is too small for additional components to be added, the parts can be built in a small plastic box that is glued or bolted to the calculator case. Space can be saved by soldering directly to the NE555. The capacitor $(100$ microfarads at $10 \mathrm{~V})$ is a miniature electrolytic type, and the two variable resistors are miniature trimmer potentiometers $(20,000 \mathrm{ohms}$ at .25 W). The miniature slide switch for turning the stopwatch on and off should be nearly silent in operation to insure that subjects will not be disturbed by the operation of the stopwatch. The LED should be the same type used to determine if the photocell is adequate for the conversion.

Since the NE555 integrated circuit can operate on $6-18 \mathrm{~V}$, it is compatible with most calculator batteries. All of the components except the switch which is bolted to the calculator case can be held in place with a drop of glue. Care should be taken not to glue the rotating parts of the miniature potentiometers. Before gluing a potentiometer in place, drill a hole in the plastic case of the calculator so that a miniature screwdriver can be inserted to adjust the potentiometer. This procedure makes it possible to calibrate the stopwatch after the calculator is reassembled. If the LED and photocell are

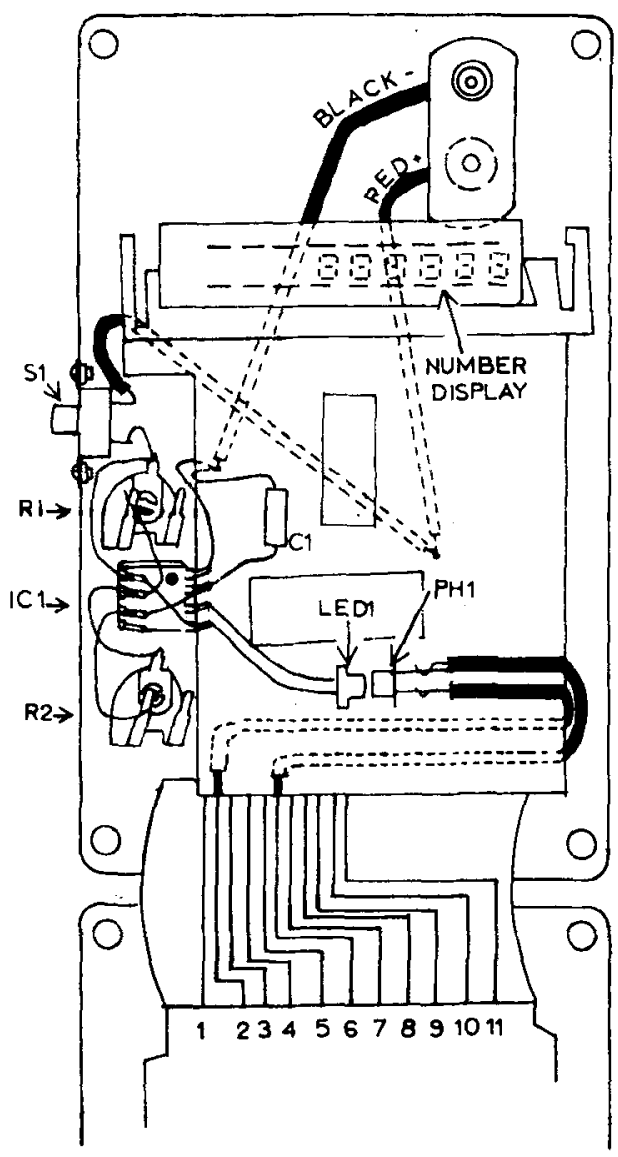

Figure 2. Pictorial diagram for stopwatch calculator. See caption for Figure 1 for component identification.

used instead of the opto-isolator, they should be glued together to insure that maximum light falls on the photocell. A piece of black tape over the photo-cell LED assembly insures that ambient light does not activate the photocell.

The calculator does not draw additional current from the battery when it is not used as a stopwatch because of the circuitry design. The timing circuit and LED draw less than $50 \mathrm{~mA}$.

Operating the stopwatch involves entering the number .1 (a " + " must also be entered on some calculators) and using the slide switch to start and stop the interval timed. If the watch is not reset by pushing the "clear" key, it can be used to cumulate times across more than one interval; the stopwatch will time to the nearest tenth of a second. The calculator can be calibrated by operating the stopwatch calculator and a standard stopwatch at the same time. The miniature potentiometers, which control pulse width and time between pulses are turned until they produce 10 pulses per second. (It is easier to calibrate the stopwatch if it is allowed to run for $30 \mathrm{sec}$ during each attempt at calibration. The stopwatch should read exactly 30.0 at the end of $30 \mathrm{sec}$ ). When the stopwatch switch is in the "off" position, the 
stopwatch calculator functions as an ordinary calculator.

If .0016667 (i.e., $.1 \mathrm{sec} / 60 \mathrm{sec}$ ) is entered in the stopwatch calculator instead of .1 , the stopwatch feature cumulates in minutes and fractions of a minute. If .0000277 (i.e., $.1 \mathrm{sec} / 60 \times 60 \mathrm{sec}$ ) is entered, the stopwatch feature cumulates in hours and fractions of an hour.

It is possible to correct for slight inaccuracies in calibrating the stopwatch. If the stopwatch is a little fast, entering .0995 instead of .1 may result in very accurate timing.

\section{REFERENCES}

Alexandrovich, Sr., G. Convert your pocket calculator into a programmable counter. Electronic Design, 1975, 23, 100.

Wolach, A. H., Roccaforte, P., \& Breuning, S. E. Converting an electronic calculator into a counter. Behavior Research Methods \& Instrumentation, 1975, 7, 365-367.

(Received for publication May 6, 1975; revision received July 7,1975 .) 